07

\title{
Светоиндуцированный флексоантиферромагнитный эффект в центроантисимметричных антиферромагнетиках
}

\author{
(C) А.Ф. Кабыченков, Ф.В. Лисовский \\ Фрязинский филиал Института радиотехники и электроники им. В.А. Котельникова РАН, \\ 141190 Фрязино, Россия \\ e-mail: lisovsky.f@yandex.ru
}

Поступило в Редакцию 14 октября 2021 г.

В окончательной редакции 28 ноября 2021 r.

Принято к публикации 18 декабря 2021 г.

Показано, что неоднородная циркулярно поляризованная световая волна индуцирует компоненты вектора антиферромагнетизма в центроантисимметричных антиферромагнетиках; узкий луч света может создавать скирмион. Выполнен анализ возможности практического использования предсказанных эффектов.

Ключевые слова: оптомагнитный эффект, полигармоническое световое поле, светоиндуцированное магнитное поле, центроантисимметричный антиферромагнетик, циркулярно поляризованная световая волна, флексоантиферромагнитный эффект.

DOI: 10.21883/JTF.2022.03.52140.276-21

Благодаря нелинейному магнитоэлектрическому эффекту световые волны в магнитоупорядоченных средах обладают способностью создавать постоянное эффективное магнитное поле, а также изменять обменное поле, поле анизотропии и намагниченности подрешеток [1-5]. С квантовой точки зрения световое поле изменяет энергетический спектр (нелинейный эффект Штарка) и степень заполнения энергических уровней, что приводит к изменению обменного и спин-орбитального взаимодействия и, следовательно, к изменению величины и направления векторов намагниченности. Согласно принятой в настоящее время терминологии, различают оптомагнитные [4] и фотомагнитные [6] эффекты. Первые проявляются в области прозрачности магнетика, а вторые в области поглощения, где электроны под действием света забрасываются в зону проводимости и на локальные энергетические уровни. Оптомагнитные эффекты, вызываемые виртуальными возбуждениями электронов, характеризуются малым временем релаксации и требуют для наблюдения большой интенсивности света, в отличие от фотомагнитных, которые имеют большие времена релаксации и наблюдаются при гораздо меньшей интенсивности света.

Учет магнитной симметрии позволил предсказать и обнаружить новые явления, заключающиеся в способности световых волн создавать магнитоэлектрические, магнитострикционные, магнетокалорические, гальваномагнитные и термогальваномагнитные эффекты, индуцировать спонтанные и ориентационные фазовые переходы первого и второго рода, производить диссипативные потоки и сопряженные им обобщенные силы [4-10].

Светоиндуцированные (СИ) магнитные поля могут производить заметные изменения ориентации векторов намагниченности подрешеток [3-16], причем вблизи точек потери статической и (или) динамической устойчи- вости, например при фазовых переходах эти изменения будут значительными, а в области гистерезиса они могут запоминаться.

Полигармоническое световое поле создает как постоянные, так переменные эффективные поля [5]. Если разность частот гармоник СИ переменных полей попадает в область ферромагнитного или антиферромагнитного резонанса, то возбуждается прецессия намагниченности и наблюдаются динамические фазовые переходы или спиновое эхо [8]. Волновые числа СИ переменных полей зависят от направления распространения гармоник и лежат в широких пределах от суммы и до разностей волновых чисел для попутных и встречных гармоник соответственно. Скорость таких СИ волн может превышать скорость спиновых волн при антиферромагнитном резонансе, что может быть использовано для их усиления и для генерирования когерентного излучения терагерцового диапазона.

СИ магнитные поля имеют преимущества по сравнению с „обычными“ магнитными полями и полями упругих напряжений, поскольку они локализованы в пределах светового луча, допускают реализацию в виде волнового пакета малой длительности, не создают электромагнитных помех, в зависимости от поляризации СИ поля проявляются как магнитное поле или упругие напряжения, могут быть постоянными и переменными на частотах в области антиферромагнитного резонанса [5].

В технике оптомагнитные эффекты в антиферромагнетиках могут использоваться для записи информации в области магнитного гистерезиса (в частности, в окрестности однородного и неоднородного магнитного фазового перехода 1-го рода), для создания и уничтожения цилиндрических магнитных доменов, для управления блоховскими линиями, для усиления спиновых волн, для создания локальных магнитных потенциалов 
заданного размера (в резонаторах, волноводах, решетках, оптомагнитных „пинцетах“ и т.д.), в том числе сравнимого с длиной волны света, для создания спинполяризованного электрического тока. Быстродействие многих из перечисленных выше операций может быть доведено до полупериода свободной прецессии, что для антиферромагнетиков составляет около $10^{-11} \mathrm{~s}$.

Распространение света в магнетиках представляет собой самосогласованный процесс, поскольку световая волна изменяет векторы намагниченности подрешеток, что, в свою очередь, влияет на параметры световой волны. В частности, могут наблюдаться магнитооптические солитоны, а также самофокусировка и возникновение магнитооптических каналов [5], поэтому оптомагнитные эффекты обнаруживаются при коротких длинах пробега света, где амплитуда и поляризация волн изменяются незначительно.

В настоящей работе СИ оптофлексоантиферромагнитный эффект рассматривается по аналогии с флексоантиферромагнитным $[17,18]$.

Средняя по времени плотность энергии среды в световом поле при слабом поглощении и низкой временной дисперсии представляется в виде

$$
w_{m l}=(1 / 16 \pi) \varepsilon_{i j} E_{i} E_{j}^{*},
$$

где $\varepsilon_{i j}-$ компоненты тензора диэлектрической проницаемости, а $E_{i}$ - компоненты комплексного электрического поля световой волны [19].

Выделяя симметричные и антисимметричные компоненты, находим, что

$$
w_{m l}=\varepsilon_{i j}^{(s)} T_{i j}^{(s)}-\mathbf{g G}
$$

где $\varepsilon_{i j}^{(s)}$ представляет собой симметричную часть тензора диэлектрической проницаемости, $\quad T_{i j}^{(s)}=(1 / 32 \pi)\left(E_{i} E_{j}^{*}+E_{i}^{*} E_{j}\right)-$ симметричную часть тензора „светового напряжения“, $\mathbf{g}$ - вектор гирации, $\mathbf{G}=(i / 16 \pi)\left[\mathbf{E}^{*} \mathbf{E}\right]-$ эффективное „магнитное поле“. Учитывая то, что в общем случае для антиферромагнетика

$$
\begin{gathered}
g_{k}=\alpha_{k l} L_{l}+\alpha_{k l}^{\prime} M_{l}+\eta_{k l m}^{(l)} \frac{\partial L_{l}}{\partial x_{m}}+\eta_{k l m}^{(m)} \frac{\partial M_{l}}{\partial x_{m}} \ldots, \\
\varepsilon_{i j}^{(s)}=\varepsilon_{i j}^{(0)}+\beta_{i j k l} L_{k} L_{l}+\beta_{i j k \ln }^{(1)} L_{k} \frac{\partial L_{l}}{\partial x_{n}}+\beta_{i j k l}^{(2)} M_{k} L_{l} \\
+\beta_{i j l \ln }^{(3)} M_{k} \frac{\partial L_{l}}{\partial x_{n}}+\beta_{i j k l}^{(4)} M_{k} M_{l},
\end{gathered}
$$

где $\mathbf{L}$ и $\mathbf{M}$ - векторы антиферромагнетизма и ферромагнетизма, находим, что в центроантисимметричных антиферромагнетиках с симметрией $\overline{3}^{\prime} m^{\prime}$ (типа $\mathrm{Cr}_{2} \mathrm{O}_{3}$ ) выражение (2) сводится к виду

$$
\begin{aligned}
& w_{m l}=-\eta_{1}^{(l)}\left[+\left(\frac{\partial L_{x}}{\partial x}-\frac{\partial L_{y}}{\partial y}\right) G_{x}+\left(\frac{\partial L_{x}}{\partial y}-\frac{\partial L_{y}}{\partial x}\right) G_{y}\right] \\
& -\eta_{2}^{(l)}\left(\frac{\partial L_{y}}{\partial z} G_{x}-\frac{\partial L_{x}}{\partial z} G_{y}\right)-\eta_{3}^{(l)}\left(\frac{\partial L_{z}}{\partial x} G_{y}-\frac{\partial L_{y}}{\partial z} G_{x}\right) \\
& -\eta_{4}^{(l)}\left(\frac{\partial L_{x}}{\partial y}-\frac{\partial L_{y}}{\partial x}\right) G_{z}-\alpha_{1}^{\prime}\left(M_{x} G_{x}+M_{y} G_{y}\right) \\
& -\alpha_{3}^{\prime} M_{z} G_{z}+\beta_{12} T_{i i}^{(s)} L^{2}+\left(\beta_{11}-\beta_{22}\right)\left(T_{11}^{(s)} L_{x}^{2}+T_{22}^{(s)} L_{y}^{2}\right. \\
& \left.+T_{33}^{(s)} L_{z}^{2}\right)+\beta_{44}\left(T_{23}^{(s)} L_{y} L_{z}+T_{31}^{(s)} L_{z} L_{x}+T_{12}^{(s)} L_{x} L_{y}\right) .
\end{aligned}
$$

По соображениям симметрии отсутствуют первое и четвертое слагаемые в (3) и третье и четвертое в (4), а два последних слагаемые в (4) не учитываются из-за малости по сравнению со вторым.

Полная плотность энергии антиферромагнетика для безграничной среды в отсутствие внешнего магнитного поля дается выражением $w=w_{0}+w_{m}+w_{m l}$, где $w_{0}$ энергия основного однородного состояния,

$$
\begin{aligned}
w_{M}= & (1 / 2)\left[a_{1}^{(l)} \mathbf{L}^{2}+a_{1}^{(m)} \mathbf{M}^{2}+a_{1}^{(\ln )}\left(\partial \mathbf{L} / \partial x_{i}\right)^{2}\right. \\
& \left.+a_{2}^{(l)} L_{z}^{2}+a_{2}^{(m)} M_{z}^{2}+a_{2}^{(\ln }\left(\partial L_{z} / \partial x_{i}\right)^{2}\right] \\
& -\eta_{1}^{(m)}\left[M_{x}\left(\frac{\partial L_{x}}{\partial x}-\frac{\partial L_{y}}{\partial y}\right)-M_{y}\left(\frac{\partial L_{x}}{\partial y}+\frac{\partial L_{y}}{\partial x}\right)\right] \\
& -\eta_{2}^{(m)}\left(M_{x} \frac{\partial L_{y}}{\partial z}-M_{y} \frac{\partial L_{x}}{\partial z}\right)-\eta_{3}^{(m)}\left(M_{y} \frac{\partial L_{z}}{\partial x}\right. \\
& \left.-M_{x} \frac{\partial L_{z}}{\partial y}\right)-\eta_{4}^{(m)} M_{z}\left(\frac{\partial L_{x}}{\partial y}-\frac{\partial L_{y}}{\partial y}\right)
\end{aligned}
$$

- энергия, вызванная внешними полями в квадратичном приближении.

Из (5) и (6) следует, что световое поле смещает точку Нееля, перенормирует константы анизотропии, создает эффективное магнитное поле, поле анизотропии и приводит к существованию оптофлексоантиферромагнитного эффекта.

Потребовав, чтобы $\delta w / d \mathbf{M}=0, \delta w / d \mathbf{L}=0$, находим уравнения состояния

$$
\begin{gathered}
M_{x}=\eta_{1}^{(m) \prime}\left(\frac{\partial L_{x}}{\partial x}-\frac{\partial L_{y}}{\partial y}\right)+\eta_{2}^{(m)}, \frac{\partial L_{y}}{\partial z}-\eta_{3}^{(m)} \frac{\partial L_{z}}{\partial y}+\chi_{\perp}^{(l)} G_{x}, \\
M_{y}=-\eta_{1}^{(m) \prime}\left(\frac{\partial L_{x}}{\partial y}+\frac{\partial L_{y}}{\partial x}\right)-\eta_{2}^{(m) \prime} \frac{\partial L_{x}}{\partial z}+\eta_{3}^{(m) \prime} \frac{\partial L_{z}}{\partial x}+\chi_{\perp}^{(l)} G_{y} \\
M_{z}=\eta_{4}^{(m) \prime}\left(\frac{\partial L_{x}}{\partial y}-\frac{\partial L_{y}}{\partial x}\right)+\chi_{\|}^{(l)} G_{z}, \\
\alpha_{11}^{(l)} L_{x}+a_{12}^{(l)} L_{y}+a_{13}^{(l)} L_{z}-a_{1}^{(\mathrm{ln})} \Delta L_{x}= \\
-\eta_{1}^{(m)}\left(\frac{\partial M_{x}}{\partial x}-\frac{\partial M_{y}}{\partial y}\right)-\eta_{4}^{(m)} \frac{\partial M_{z}}{\partial y}+\eta_{2}^{(m)} \frac{\partial M_{y}}{\partial z} \\
-\eta_{1}^{(l)}\left(\frac{\partial G_{x}}{\partial x}-\frac{\partial G_{y}}{\partial y}\right)-\eta_{4}^{(l)} \frac{\partial G_{z}}{\partial y}+\eta_{2}^{(l)} \frac{\partial G_{y}}{\partial z}
\end{gathered}
$$




$$
\begin{gathered}
\alpha_{12}^{(l)} L_{x}+a_{22}^{(l)} L_{y}+a_{13}^{(l)} L_{z}-a_{1}^{(\mathrm{ln})} \Delta L_{y}= \\
\eta_{1}^{(m)}\left(\frac{\partial M_{x}}{\partial y}+\frac{\partial M_{y}}{\partial x}\right)+\eta_{4}^{(m)} \frac{\partial M_{z}}{\partial x}-\eta_{2}^{(m)} \frac{\partial M_{x}}{\partial z} \\
+\eta_{1}^{(l)}\left(\frac{\partial G_{x}}{\partial y}+\frac{\partial G_{y}}{\partial x}\right)+\eta_{4}^{(l)} \frac{\partial G_{z}}{\partial x}-\eta_{2}^{(l)} \frac{\partial G_{x}}{\partial z}, \\
\alpha_{13}^{(l)} L_{x}+a_{22}^{(l)} L_{y}+a_{33}^{(l)} L_{z}-a_{12}^{(\mathrm{ln})} \Delta L_{z}= \\
\eta_{3}^{(m)}\left(\frac{\partial M_{x}}{\partial y}-\frac{\partial M_{y}}{\partial x}\right)+\eta_{3}^{(l)}\left(\frac{\partial G_{x}}{\partial y}-\frac{\partial G_{y}}{\partial x}\right), \\
\eta_{n}^{(m) \prime}=\eta_{n}^{(m)} / a_{1}^{(m)}, \chi_{\perp}=1 / a_{1}^{(m)}, \chi_{\perp}^{(l)}=\alpha_{1}^{\prime} / a_{1}^{(m)}, \\
\chi_{\|}=1 /\left(a_{1}^{(m)}+a_{2}^{(m)}\right), \chi_{\|}^{(l)}=\alpha_{3}^{\prime} /\left(a_{1}^{(m)}+a_{2}^{(m)}\right), \\
a_{\mu}^{(l)}=a_{1}^{(l)}+\delta_{3 i} a_{2}^{(l)}+2 \beta_{12} T_{i i}^{(s)}+2\left(\beta_{11}-\beta_{12}\right) T_{\mu}^{(s)}, \\
\mu=11,22,33, a_{i j}^{(l)}=\beta_{44} T_{i j}^{(s)},
\end{gathered}
$$

где $a_{12}^{(\ln )}=a_{1}^{(\ln )}+a_{2}^{(\ln )}$.

Видно, что возникновение намагниченности в рассматриваемом случае обусловлено неоднородностями антиферромагнитного вектора, т.е. имеет место флексоантиферромагнитный эффект, который отличается от слабого ферромагнетизма, при котором намагниченность существует при однородном распределении антиферромагнитного вектора $[20,21]$.

$\mathrm{B}$ центроантисимметричных антиферромагнетиках слабый ферромагнетизм отсутствует и флексоантиферромагнитный эффект не маскируется. Циркулярно поляризованная компонента светового поля наводит намагниченность $(M \propto G)$, а линейно поляризованная изменяет модуль и направление вектора антиферромагнетизма (оптомагнитный эффект). Кроме того, неоднородная циркулярно поляризованная компонента света наводит компоненты вектора антиферромагнетизма $L_{i}=n_{i j k} \partial G_{j} / \partial x_{K}$, т. е. имеет место оптофлексоантиферромагнитный эффект. Если учитывать малые слагаемые в (4), то неоднородности линейно поляризованного светового поля также будут наводить компоненты антиферромагнитного вектора.

Подстановка выражений для $M_{i}$ в три последние уравнения системы (7) дает возможность определить и компоненты $L_{i}$. В отсутствие внешнего магнитного поля, связанные с М слагаемые будут пропорциональны квадратам констант $\eta_{i}^{(m)} \eta_{j}^{(m)}$ и $\eta_{i}^{(m)} \alpha_{j}^{\prime}$, и первом приближении их можно не учитывать. Кроме того, на размерах порядка длины волны света будут пренебрежимо малыми и слагаемые, обусловленные неоднородным обменным взаимодействием. В этом случае для циркулярно поляризованной волны, распространяющейся вдоль оси симметрии с $E_{y}=-i E_{x}$ имеем

$$
L_{x}=-\left(\eta_{4}^{l} / a_{11}^{(l)}\right) \frac{\partial G_{z}}{\partial y}, L_{y}=\left(\eta_{4}^{l} / a_{11}^{(l)}\right) \frac{\partial G_{z}}{\partial x}, L_{z}=0,
$$

где

$$
a_{11}^{(l)}=a_{1}^{(l)}+\left[\left(\beta_{11}+\beta_{12}\right)\left|E_{x}\right|^{2} / 16 \pi\right], \quad G_{z}=\left|E_{x}\right|^{2} / 8 \pi .
$$

В световом луче напряженность электрического поля имеет максимум на оси и спадает до нуля при удалении от нее. Следовательно, компоненты $L_{x}$ и $L_{y}$ исчезают на оси и вне луча, достигая максимальных значений в точках с наибольшим градиентом напряженности светового поля, образуя скирмион. Световое поле с гауссовым распределением $\left|E_{x}\right|^{2}=|E|_{0}^{2} \exp \left(-\rho^{2} / \rho_{0}^{2}\right)$ индуцирует компоненту $L_{\varphi}$, направленную по касательной к окружности и имеющую модуль

$$
L_{\varphi}\left(\eta_{4}^{(l)} /\left|a_{11}^{(l)}\right|\right)\left(|E|_{0}^{2} 8 \pi\right)\left(2 \rho / \rho_{0}^{2}\right) \exp \left(-\rho^{2} / \rho_{0}^{2}\right)
$$

где $\rho^{2}=x^{2}+y^{2}$.

Линейная по материальной константе компонента намагниченности определяется выражением $M_{z}=\chi_{\|}^{(l)} G_{z}$. Используя (8), из первого и второго уравнений системы (7) можно определить значения $M_{x}$ и $M_{y}$, подстановка которых в третье уравнение системы (7) позволяет найти во втором приближении величину и другие СИ компоненты

$$
L_{z}=c_{0}\left[8 x\left(3 y^{2}-x^{2}\right) / \rho_{0}^{6}\right] \exp \left(-\rho^{2} / \rho_{0}^{2}\right),
$$

где

$$
c_{0}\left(\eta_{3}^{(m)} \eta_{1}^{(m)} \eta_{4}^{(l)} / a_{1}^{(l)} a_{1}^{(m)} a_{3}^{(l)}\right)\left|E_{0}\right|^{2} / 8 \pi .
$$

Заметим, что пучки света могут создавать различные топологические магнитные особенности. В частности, это относится к циркулярно поляризованному световому полю, которое перенормирует константу однородного обмена, создает дополнительные эффективное магнитное поле, легкоосевую или легкоплоскостную анизотропию. Если в легкоплоскостном или легкоосевом магнетике свет наводит легкоосевую или легкоплоскостную СИ анизотропию, то в пучке векторы намагниченности выходят из плоскости или отклоняются от оси, образуя магнитный вихрь, который может содержать разные фазы [4]. Спиральный пучок света создает скирмион [22]. В основе этих магнитных особенностей лежат известные эффекты, в отличие от описанного в настоящей работе скирмиона, обусловленного оптофлексоантиферромагнитным эффектом.

Главным результатом настоящей работы можно считать теоретическое обоснование того, что воздействие неоднородных циркулярно или линейно поляризованных световых волн на центроантисимметричные антиферромагнетики индуцирует возникновение ряда ранее неизвестных эффектов, которые могут быть использованы и для дефектоскопии, и для управляемого изменения параметров таких сред, которые находят широкое использование в спинтронике $[23,24]$. Управление светом имеет несомненные преимущества перед другими методами, поскольку является локальным, дистанционным и сохраняет эффективность даже при использовании ультракоротких импульсов $[13,14]$. Следует, однако, иметь в виду, что улучшение такого способа путем увеличения интенсивности света приводит к возрастанию побочных эффектов, в частности, к нагреву магнетика. Поэтому в 
настоящее время изыскиваются альтернативные способы усиления эффективности оптомагнитных эффектов, в частности, обратного эффекта Фарадея [25].

Практически полное отсутствие необходимых экспериментальных данных исключает в настоящее время возможность численной оценки материальных констант, входящих в уравнение (3) и определяющих величину флексоантиферромагнитного эффекта и возможность его обнаружения. Обнадеживающим, однако, является то обстоятельство, что авторы работ [26,27], наблюдавшие новые невзаимные оптические явления в антиферромагнетике $\mathrm{Cr}_{2} \mathrm{O}_{3}$ в электрическом $\mathbf{E}_{\mathrm{O}}$ поле, связывали возможность их существования с наличием в гиротропной части плотности энергии среды слагаемого $\eta_{k l m}^{(e)} L_{l} E_{m}^{(0)}$ которое по структуре и симметрии совпадает с третьим членом уравнения (3), а именно $\eta_{k l m}^{(l)} \frac{\partial L_{l}}{\partial x_{m}}$. При использовании узких пучков света градиент $\mathbf{L}$ может создавать соразмерный с $\mathbf{E}_{O}$ эффект.

\section{Финансирование работы}

Работа выполнена в рамках государственного задания Института радиотехники и электроники им. В.А. Котельникова РАН.

\section{Конфликт интересов}

Авторы заявляют, что у них нет конфликта интересов.

\section{Список литературы}

[1] Л.П. Питаевский. ЖЭТФ, 39 (5), 1450 (1960).

[2] P.S. Pershan, I.P. van der Ziel, L.D. Malmstrom. Phys. Rev., 143 (2), 574 (1966).

[3] А.М. Балбашов, Б.А. Зон, В.Я. Купершмидт, Г.В. Пахомов, Т.Т. Уразбаев. ЖЭТФ, 94 (5), 304 (1988).

[4] А.Ф. Кабыченков. ЖЭТФ, 100 (10), 1219 (1991).

[5] A.F. Kabychenkov. In „Studies in Applied Electromagnetics and Mechanics", 13, Nonlinear Electromagnetic System, ed. by V. Kose, J. Sievert (1998), c. 879-882.

[6] В.Ф. Коваленко, Э.Л. Нагаев. УФН, 148 (4), 561 (1986).

[7] А.Ф. Кабыченков. Письма ЖТФ, 33 (1), 45 (2007).

[8] А.Ф. Кабыченков. ФТТ, 48 (3), 485 (2006).

[9] А.Ф. Кабыченков. ФТТ, 34 (12), 3781 (1992).

[10] A.F. Kabychenkov. EMSA 2000, Book of Abstracts, 285-286 (2000).

[11] A.V. Kimel, A. Kirilyuk, P.A. Usachev, R.V. Pisarev, A.M. Balbashov, Th. Rasing. Nature, 435, 655 (2005).

[12] A.V. Kimel, B.A. Ivanov, R.V. Pisarev, P.A. Usachev, A. Kirilyuk, Th. Rasing. Nature Physics, 5, 727 (2009).

[13] A. Kirilyuk, A.V. Kimel, T. Rasing. Rev. Mod. Phys., 82, 2731 (2010).

[14] Б.А. Иванов. ФНТ, 40 (2), 119 (2014).

[15] D. Afanasiev, B.A. Ivanov, A. Kirilyuk, Th. Rasing, R.V. Pisarev, A.V. Kimel. Phys. Rev. Lett., 116, 097401 (2016).

[16] А.М. Калашникова, А.В. Киммель, Р.В. Писарев. УФН, 185 (10), 1064 (2005).
[17] А.Ф. Кабыченков, Ф.В. Лисовский. ЖЭТФ, 145 (4), 733 (2014).

[18] А.Ф. Кабыченков, Ф.В. Лисовский. ЖТФ, 89(7), 1039 (2019).

[19] Л.Д. Ландау, Е.М. Лифшиц. Электродинамика сплошных сред (Наука, М., 1992)

[20] И.Е. Дзялошинский. ЖЭТФ, 32 (6), 1547 (1957).

[21] Е.А. Туров, А.В. Колчанов, В.В. Меньшенин, И.Ф. Мирсаев, В.В. Николаев. Симметрия и физические свойства антиферромагнетиков (Физматлит, М., 2001)

[22] H. Fujita, M. Sato. Phys. Rev. B, 95, 054421 (2017).

[23] Н.Н. Крупа, И.В. Шарай. Успехи физ. мат., 14, 1 (2013).

[24] Ю.К. Фетисов, Ф.С. Сигов. РЭНСИТ, 10 (3), 343 (2018).

[25] Е.А. Безус, В.И. Белотелов, Л.Л. Доскалович, А.К. Звездин. Компьютерная оптика, 35 (4), 431 (1011).

[26] Б.Б. Кричевцов, В.В. Павлов, Р.В. Писарев. Письма в ЖЭТФ, 44 (10), 471 (1986).

[27] Б.Б. Кричевцов, В.В. Павлов, Р.В. Писарев. ЖЭТФ, 94 (10), 284 (1988). 